\title{
Mutation and linkage disequilibrium analysis in genetic counselling of Spanish cystic fibrosis families
}

\author{
T Casals, V Nunes, C Lázaro, F J Giménez, E Girbau, V Volpini, X Estivill
}

\begin{abstract}
We have analysed haplotypes for four DNA polymorphisms, closely linked to the cystic fibrosis (CF) gene, in 82 Spanish families, in which the CF probands are either homozygous for non- $\Delta F 508$ mutations or heterozygous for the $\Delta F 508$ deletion and other CF mutations. The analysis provides genetic data for a new polymorphism for the closely linked marker pKM.19, which is very strongly associated with CF. Haplotypes generated with the four marker loci are also in strong disequilibrium with the non- $\Delta F 508$ CF chromosomes. The data reported here are useful in 1 in 4 risk pregnancies of parents who have no living affected child, and when counselling close relatives of CF families who are negative for the major CF mutation. The data presented are useful in our population, in which the majority of CF mutations, apart from the $\Delta F 508$ deletion, are uncommon. For other populations in which mutation heterogeneity is also very high, it still might be more feasible to use RFLPs for diagnostic purposes, when analysis for common mutations is negative and DNA is available from the index patient. The experience presented here provides a model for these population groups who in turn should obtain their own haplotype data. In addition, the model system for genetic counselling presented here might also be useful for other genetic disorders.
\end{abstract}

Molecular Genetics Department, Cancer Research Institute, Hospital Duran i Reynals, Barcelona, Catalonia, Spain.

T Casals, V Nunes, C Lázaro, F J Giménez, E Girbau, V Volpini, X Estivill

Correspondence to Dr Estivill, Department Genética Molecular, Institut de Recerca Oncológica, Hospital Duran i Reynals, L'Hospitalet de Llobregat, 08907 Barcelona, Catalonia, Spain.

Received for publication 22 January 1991.

Revised version accepted for publication 8 April 1991.
Genetic testing for cystic fibrosis (CF) has been considerably improved since the isolation of the CF gene and the discovery of the major CF mutation, which consists of a three nucleotide deletion $(\Delta \mathrm{F} 508)$ in the first putative ATP binding domain of the predicted protein (CF transmembrane conductance regulator, CFTR). ${ }^{1-3}$

The $\Delta$ F508 deletion has been found in approximately $70 \%$ of North American ${ }^{34}$ and north European ${ }^{5}$ CF chromosomes, but in only $50 \%$ of south Europeans. ${ }^{67}$ Although several other mutations have been identified (CF Genetic Consortium), most of them are very uncommon, ${ }^{8-10}$ and others have a frequency of approximately $3.5 \%$ in North Americans and north Europeans, but they seem to be even rarer in other populations. ${ }^{8}$

Haplotype analysis of CF chromosomes based on DNA markers closely linked to the disease locus suggests that the remainder of the CF mutant allele pool (non- $\Delta$ F508 mutations) in the Spanish population consists of various distinct mutations. ${ }^{311}$ Mutation analysis provides, at the moment, full informativeness for prenatal diagnosis in only $25 \%$ (south European) to $55 \%$ (north European and North American) of couples. ${ }^{42}$ Therefore, prenatal diagnosis and carrier detection of $\mathrm{CF}$ is currently performed by the analysis of the $\Delta \mathrm{F} 508$ deletion or other defined mutations, and by using closely linked markers detecting RFLPs. ${ }^{42}$ As the non- $\Delta$ F508 CF mutant allele pool is so heterogeneous, it may be more useful to use closely linked markers for genetic diagnosis of $\mathrm{CF}$, when mutation analysis is negative for the common mutations.

We present here the haplotype analysis of $\Delta \mathrm{F} 508$, non- $\Delta$ F508 CF chromosomes, and normal chromosomes, in CF families of Spanish origin, with four RFLPs closely linked to CF. A new RFLP analysed, pKM.19/Scrf I, ${ }^{13}$ shows the highest non-random allelic association with $C F$ (in both $\Delta F 508$ and non$\Delta$ F508 CF chromosomes) described so far. The association between several haplotypes and non$\Delta$ F508 chromosomes might be used to improve genetic analysis when DNA is not available from the $\mathrm{CF}$ proband and when counselling close relatives of CF families negative for common CF mutations. ${ }^{14}$ 


\section{Materials and methods}

DNA analysis was performed on a large series of families with members affected with CF. The families were referred to our centre in Barcelona between 1986 and 1989 from most of the regions of the country, and all were of Spanish origin. Diagnosis was confirmed by both the typical symptoms and two positive sweat tests. Only the families in which the CF patients were either homozygous for non$\Delta F 508$ mutations or heterozygous for the $\Delta F 508$ deletion and other unknown CF mutations (in total 82 families) were considered for the present analysis. ${ }^{11}$

Genomic DNA from blood containing EDTA as anticoagulant was extracted from the parents, the CF patient, and in some cases from the grandparents. DNA was subjected to amplification as recommended by the manufacturer of $T a q$ polymerase. Each $100 \mu \mathrm{l}$ reaction mixture contained $50 \mathrm{mmol} / \mathrm{l}$ potassium chloride, $10 \mathrm{mmol} / \mathrm{l}$ Tris-hydrochloric acid ( $\mathrm{pH} 7.8$ ), $1.5 \mathrm{mmol} / \mathrm{l}$ magnesium chloride, $200 \mu \mathrm{mol} / 1$ of each deoxynucleotide triphosphate, $30 \mathrm{pmol} / \mathrm{l}$ of each oligonucleotide primer, $300 \mathrm{ng}$ of genomic DNA, and 2.0 units of Taq polymerase; $50 \mu \mathrm{l}$ of mineral oil was added to each reaction.

We studied four polymorphic loci: $\mathrm{pXV}-2 \mathrm{c} / \mathrm{TaqI}$, pKM.19/ScrfI, pKM.19/PstI, and pMP6d-9/MspI. The loci were analysed by restriction enzyme digestion after PCR amplification with the pairs of primers previously described ${ }^{15-18}$ or by Southern blotting. Sequences for the amplification of the exon 10 region, containing the $\Delta \mathrm{F} 508$ mutation, were from Riordan et al. ${ }^{2}$ After an initial step of denaturing at $95^{\circ} \mathrm{C}$ for five minutes, 30 cycles were performed, including a 30 second denaturing step at $95^{\circ} \mathrm{C}$, a 30 second annealing step as described, and one minute of polymerisation at $72^{\circ} \mathrm{C}$. The last cycle was followed by a 10 minute step at $72^{\circ} \mathrm{C}$. After amplification, $25 \mu \mathrm{l}$ of sample was directly digested with the respective restriction enzyme. For the detection of the $\Delta \mathrm{F} 508$ mutation, $5 \mu \mathrm{l}$ of formamidedye mixture ( $95 \%$ formamide $/ 0.05 \%$ bromophenol blue $/ 0.05 \%$ xylene cyanol $/ 20 \mathrm{mmol} / 1$ EDTA) were added to $15 \mu$ l of the amplified DNA. Samples were loaded on to a $1 \mathrm{~mm}$ thick, $20 \mathrm{~cm} \times 20 \mathrm{~cm} \%$ PAGE $(1.6 \mathrm{~mol} / 1$ urea) in $1 \times \mathrm{TBE}$ buffer. Electrophoresis was performed at $400 \mathrm{~V}$ for two hours. Fragments of either 95 bp $(\Delta F 508)$ or 98 bp were directly visualised using an UV transilluminator.

The degree of association between DNA markers and CF was measured by Yule's association coefficient. The standardised association $(A)=(a d-b c) /$ $(a d+b c)$, where $a, b, c$, and $d$ are the numbers of normal chromosomes with allele 1, CF non- $\Delta$ F508 with 1 , normal with 2 , and CF non- $\Delta$ F508 with 2 , respectively. ${ }^{3}$

\section{Results and discussion}

Since the $\Delta$ F508 mutation accounts for only $50 \%$ of the Spanish CF chromosomes, and other mutations in the gene are very uncommon, the aim of this study was to evaluate the power of haplotype analysis when applied to genetic counselling and to provide more accurate carrier risk figures.

The results of haplotype analysis for $\mathrm{pXV}-2 \mathrm{c}$, pKM.19, and pMP6d-9 in the 82 Spanish CF families in which the index patients are either homozygous for non- $\Delta$ F508 mutations or heterozygous for the $\Delta$ F508 deletion and another CF mutation are presented in table 1 . The four loci analysed are situated at the $5^{\prime}$ end of the CF gene' in the following order: pXV-2c/TaqI, pKM.19/ScrfI, pKM.19/ Pst I, and pMP6d-9/MspI. Eleven haplotypes were found in total.

Haplotype data can be used to improve genetic counselling in the following situations: (1) couples

Table 1 Haplotype data for 82 Spanish CF families not homozygous or heterozygous for the $\triangle F 508$ mutation.

\begin{tabular}{|c|c|c|c|c|c|c|c|c|c|c|}
\hline \multirow[b]{3}{*}{ Haplotype } & \multirow[b]{3}{*}{$\mathrm{T}$} & \multirow[b]{3}{*}{$\mathbf{S}$} & \multirow[b]{3}{*}{$\mathbf{P}$} & \multirow[b]{3}{*}{$\mathbf{M}$} & \multicolumn{6}{|c|}{ Chromosomes } \\
\hline & & & & & \multicolumn{2}{|c|}{ CF (non- $\Delta$ F508) } & \multicolumn{2}{|c|}{ Normal } & \multicolumn{2}{|c|}{$\mathrm{CF}(\Delta \mathrm{F} 508)$} \\
\hline & & & & & No & $\%$ & No & $\%$ & No & $\%$ \\
\hline $\begin{array}{l}\mathbf{a} \\
\mathbf{b} \\
\mathbf{c} \\
\mathbf{d} \\
\mathbf{e} \\
\mathbf{f} \\
\mathbf{g} \\
\mathbf{h} \\
\mathbf{i} \\
\mathbf{j} \\
\mathbf{k}\end{array}$ & $\begin{array}{l}1 \\
2 \\
2 \\
1 \\
1 \\
1 \\
1 \\
2 \\
2 \\
2 \\
2\end{array}$ & $\begin{array}{l}1 \\
1 \\
1 \\
2 \\
2 \\
2 \\
1 \\
2 \\
2 \\
2 \\
2\end{array}$ & $\begin{array}{l}2 \\
2 \\
1 \\
1 \\
2 \\
1 \\
1 \\
2 \\
1 \\
1 \\
2\end{array}$ & $\begin{array}{l}2 \\
2 \\
1 \\
2 \\
2 \\
1 \\
1 \\
2 \\
1 \\
2 \\
1\end{array}$ & $\begin{array}{r}39 \\
2 \\
0 \\
0 \\
11 \\
21 \\
0 \\
7 \\
30 \\
4 \\
0\end{array}$ & $\begin{array}{r}34.2 \\
1.8 \\
0.0 \\
0.0 \\
9.7 \\
18.4 \\
0.0 \\
6.1 \\
26.3 \\
3.5 \\
0.0\end{array}$ & $\begin{array}{r}7 \\
1 \\
1 \\
5 \\
13 \\
47 \\
1 \\
26 \\
58 \\
3 \\
2\end{array}$ & $\begin{array}{r}4.3 \\
0.6 \\
0.6 \\
3.0 \\
7.9 \\
28.7 \\
0.6 \\
15.9 \\
35.4 \\
1.8 \\
1.2\end{array}$ & $\begin{array}{r}47 \\
1 \\
0 \\
1 \\
0 \\
0 \\
0 \\
1 \\
0 \\
0 \\
0\end{array}$ & $\begin{array}{r}94 \cdot 0 \\
2 \cdot 0 \\
0.0 \\
2 \cdot 0 \\
0.0 \\
0.0 \\
0.0 \\
2 \cdot 0 \\
0.0 \\
0.0 \\
0.0\end{array}$ \\
\hline Total & & & & & 114 & & 164 & & 50 & \\
\hline
\end{tabular}

$\mathrm{T}=\mathrm{pXV}-2 \mathrm{c} /$ TaqI, $\mathrm{S}=\mathrm{pKM} .19 /$ ScrfI, $\mathrm{P}=\mathrm{pKM} .19 /$ PstI, $\mathrm{M}=\mathrm{pMP} 6 \mathrm{~d}-9 / M s p \mathrm{I}$. 
Table 2 Probability that a non- $\triangle F 508$ chromosome of a given haplotype is a CF chromosome $[P(n-\Delta F \mid a)]$.

\begin{tabular}{llc}
\hline Haplotype & Probability & \multicolumn{1}{c}{ Odds } \\
\hline a & 0.0749 & 1 in 13.3 \\
b & 0.0282 & 1 in 35.5 \\
c & 0.0 & - \\
d & 0.0 & - \\
e & 0.0121 & 1 in 82.6 \\
f & 0.0064 & 1 in 156.2 \\
g & 0.0 & - \\
h & 0.00390 & 1 in 256.4 \\
i & 0.00747 & 1 in 133.9 \\
j & 0.0190 & 1 in 52.6 \\
k & 0.0 & - \\
\hline
\end{tabular}

with less than a 1 in 4 risk, and (2) couples with a 1 in 4 risk and a dead CF child, who is negative for the $\Delta$ F508 mutation.

\section{HAPLOTYPE ANALYSIS IN COUPLES WITH LESS THAN 1} IN 4 RISK

The probability that a chromosome of a given haplotype (for example, haplotype a) is a non- $\Delta$ F508 CF chromosome $[P(n-\Delta F \backslash a)]$ can be calculated from the data in table 1. This probability is based on a Bayesian calculation, and takes into account the fact that the haplotype is found in a phenotypically normal subject:

$$
P(n-\Delta F \mid a)=\frac{F_{a} \times F_{n-\Delta F}}{F_{a} \times F_{n-\Delta F}+F_{a}^{\prime}}
$$

where $F_{a}$ is the frequency of haplotype $a$ in the non$\Delta F 508 \mathrm{CF}$ chromosomes, $\mathrm{F}_{\mathrm{n}-\Delta \mathrm{F}}$ is the gene frequency of the non- $\Delta F 508$ mutations in the population (1/ 100 in the Spanish population), and $F^{\prime}$ is the frequency of haplotype $\mathbf{a}$ in the normal chromosomes after testing for the $\Delta \mathrm{F} 508$ mutation.

Table 2 shows the probabilities calculated for all the haplotypes found in the Spanish population. Four haplotypes (c, d, g, and k) were not found in this sample of 114 non- $\Delta$ F508 CF chromosomes; however, these haplotypes represent only $6 \%$ of the overall normal chromosomes. The probabilities that a non- $\Delta$ F508 chromosome with a given haplotype is a CF chromosome are considerably reduced in the case of haplotypes $f, h$, and $i$, with odds of 1 in $156 \cdot 2,1$ in $256 \cdot 4$, and 1 in 133.9 , respectively. For haplotypes $\mathbf{a}, \mathbf{b}, \mathbf{e}$, and $\mathbf{j}$, the probabilities of being CF chromosomes are increased to 1 in $13.3,1$ in $35.5,1$ in 82.6 , and 1 in 52.6, respectively. Thus, the probability of a haplotype derived from a parent with no family history of CF and not carrying the $\Delta$ F508 mutation can be ascertained using these data.

The probability that a phenotypically normal subject with genotype $\mathbf{a b}$ is a carrier of a non- $\Delta$ F508 mutation can be calculated as follows:

$P\left(C_{n-\Delta F} \mid a b\right)=\frac{\left(F_{b} \times F_{a}^{\prime}+F_{b}^{\prime} \times F_{a}\right) \times F_{n-\Delta F}}{\left(F_{b} \times F_{a}^{\prime}+F_{b}^{\prime} \times F_{a}\right) \times F_{n-\Delta F}+\left(F_{b}^{\prime} \times F_{a}^{\prime}\right)}$

where $F_{b}$ and $F_{b}^{\prime}$ are the frequencies of haplotype $b$ in their respective non- $\Delta \mathrm{F} 508$ and normal chromosome populations.

Table 3 shows the probabilities of carrying a non$\Delta F 508$ mutation for all 66 expected genotypes of phenotypically normal subjects in the Spanish population. Considering a carrier frequency for a non- $\Delta$ F508 mutation of 1 in 50, this figure is notably improved for some genotypes (for example, for genotypes df 1 in 155 and dh 1 in 256.4). For other genotypes the probabilities of being a carrier are considerably increased (aa 1 in $7 \cdot 2$, ab 1 in $10 \cdot 1$, and ad 1 in 13.3). Other genotypes only show slight modifications of the previous carrier risk figures.

Table 3 Probability that a person of a particular genotype is a carrier of a non- $4 F 508$ mutation

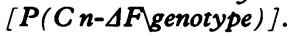

\begin{tabular}{|c|c|c|c|c|c|}
\hline Genotype & Risk & Genotype & Risk & Genotype & Risk \\
\hline $\begin{array}{l}\mathbf{a a} \\
\mathbf{a b} \\
\mathbf{a c} \\
\mathbf{a d} \\
\mathbf{a e} \\
\mathbf{a f} \\
\mathbf{a g} \\
\mathbf{a h} \\
\mathbf{a i} \\
\mathbf{a j} \\
\mathbf{a k} \\
\mathbf{b b} \\
\mathbf{b c} \\
\mathbf{b d} \\
\mathbf{b e} \\
\mathbf{b f} \\
\mathbf{b g} \\
\mathbf{b h} \\
\mathbf{b i} \\
\mathbf{b j} \\
\mathbf{b j} \\
\mathbf{c c}\end{array}$ & $\begin{array}{l}1 \text { in } 7 \cdot 2 \\
1 \text { in } 10 \cdot 1 \\
1 \text { in } 13 \cdot 3 \\
1 \text { in } 13 \cdot 3 \\
1 \text { in } 11 \cdot 7 \\
1 \text { in } 12 \cdot 4 \\
1 \text { in } 13 \cdot 3 \\
1 \text { in } 12 \cdot 8 \\
1 \text { in } 12 \cdot 3 \\
1 \text { in } 11 \cdot 0 \\
1 \text { in } 13 \cdot 3 \\
1 \text { in } 18 \cdot 2 \\
1 \text { in } 35 \cdot 5 \\
1 \text { in } 35 \cdot 5 \\
1 \text { in } 70 \cdot 4 \\
1 \text { in } 29 \cdot 1 \\
1 \text { in } 35.5 \\
1 \text { in } 31 \cdot 3 \\
1 \text { in } 28 \cdot 3 \\
1 \text { in } 21 \cdot 6 \\
1 \text { in } 35 \cdot 5 \\
\quad-\end{array}$ & $\begin{array}{l}\text { cd } \\
\text { ce } \\
\text { cf } \\
\text { cg } \\
\text { ch } \\
\text { ci } \\
\text { cj } \\
\text { ck } \\
\text { dd } \\
\text { de } \\
\text { df } \\
\text { dg } \\
\text { dh } \\
\text { di } \\
\text { dj } \\
\text { di } \\
\text { ee } \\
\text { ef } \\
\text { eg } \\
\text { eh } \\
\text { ei } \\
\text { ej }\end{array}$ & $\begin{array}{l}1 \text { in } \overline{82.6} \\
1 \text { in } 155.0 \\
1 \text { in } \overline{256.4} \\
1 \text { in } 134 \cdot 0 \\
1 \text { in } 52.6 \\
- \\
1 \text { in } \overline{82.6} \\
1 \text { in } 155 \cdot 0 \\
1 \text { in } \overline{256.4} \\
1 \text { in } 134.0 \\
1 \text { in } 52.6 \\
1 \text { in } \overline{41} \cdot 7 \\
1 \text { in } 54.3 \\
1 \text { in } 82.6 \\
1 \text { in } 62.9 \\
1 \text { in } 51.5 \\
1 \text { in } 32.6\end{array}$ & $\begin{array}{l}\text { ek } \\
\mathbf{f f} \\
\mathbf{f g} \\
\mathbf{f h} \\
\mathbf{f i} \\
\mathbf{f j} \\
\mathbf{f k} \\
\mathbf{g g} \\
\mathbf{g h} \\
\mathbf{g i} \\
\mathbf{g j} \\
\mathbf{g k} \\
\mathbf{h h} \\
\mathbf{h i} \\
\mathbf{h j} \\
\mathbf{h k} \\
\mathbf{i i} \\
\mathbf{i j} \\
\mathbf{i k} \\
\mathbf{j j} \\
\mathbf{j k} \\
\mathbf{k k}\end{array}$ & $\begin{array}{l}1 \text { in } 82.6 \\
1 \text { in } 78 \cdot 1 \\
1 \text { in } 155.0 \\
1 \text { in } 97 \cdot 1 \\
1 \text { in } 72.5 \\
1 \text { in } 39.7 \\
1 \text { in } 155.0 \\
1 \text { in } \overline{256.4} \\
1 \text { in } 134.0 \\
1 \text { in } 52.6 \\
1 \text { in } 128.9 \\
1 \text { in } 88.5 \\
1 \text { in } 44.0 \\
1 \text { in } 256.4 \\
1 \text { in } 67.6 \\
1 \text { in } 38.2 \\
1 \text { in } 134 \\
1 \text { in } 26.8 \\
1 \text { in } 52.6\end{array}$ \\
\hline
\end{tabular}


The typical situation in which haplotype information could improve the carrier risk figure is for couples with less than a 1 in 4 risk of CF (for example, couples consisting of a known carrier and a person from the general population), where the low risk parent does not have the $\Delta F 508$ mutation. The risk of the partner being a carrier is modified from 1 in 25 to 1 in 50 after mutation analysis. Haplotype analysis including the pKM.19/Scrf I marker allows the modification of their risk of being a carrier for a non- $\Delta$ F508 mutation. A final carrier risk could be given, which ranges from 1 in $7 \cdot 2$ to 1 in $256 \cdot 4$, and for some genotypes it is practically zero. Thus, for the couple, the risk of having a CF child, calculated by mutation analysis is 1 in 200 , whereas using haplotype analysis the risk can be further modified to between 1 in 28.8 and 1 in 825.6 , and in some cases to practically zero.

HAPLOTYPE ANALYSIS IN COUPLES WITH A 1 IN 4 RISK OF CF

When counselling parents of a dead child for a further pregnancy, the probabilities for CF of each possible genotype in the fetus are calculated from haplotype data in the parents using Bayes's theorem. The calculations are performed for each possible phase in each parent, under the assumption that both parents are obligate carriers of a CF mutation. Considering one parent with genotype $\mathbf{a b}$, the probabilities that the CF mutation is associated either with haplotype $\mathbf{a}$ or with haplotype $\mathbf{b}$, given the parent is $\mathbf{a b}$, are:

$$
P\left(C_{a} \backslash a b\right)=\frac{F_{a} \times F_{b}^{\prime}}{F_{a} \times F_{b}^{\prime}+F_{a}^{\prime} \times F_{b}}
$$

or

$$
P\left(C_{b} \backslash \mathbf{a b}\right)=1-P\left(C_{a} \mid a b\right)
$$

Considering that the other parent has a genotype cd, then the probabilities that the CF mutation is associated either with $\mathbf{c}$ or $\mathbf{d}$ are: $\mathrm{P}\left(\mathrm{CF}_{\mathbf{c}} \mid \mathbf{c d}\right)$ or $P\left(C F_{d} \backslash c d\right)=1-P\left(C F_{c} \backslash c d\right)$.

The probabilities for each possible genotype are the products of the probabilities that each haplotype contributed by each parent carries a CF mutation:

$$
\begin{aligned}
& \mathrm{P}\left(\mathrm{CF}_{\mathrm{ac}} \mid \mathbf{a b} \text { and } \mathrm{cd} \text { in parents }\right) \\
& =\mathrm{P}\left(\mathrm{CF}_{\mathrm{a}} \mid \mathbf{a b}\right) \times \mathrm{P}\left(\mathrm{CF}_{\mathrm{c}} \mid \mathbf{c d}\right) \\
& \mathrm{P}\left(\mathrm{CF}_{\mathrm{bc}} \mid \mathrm{ab} \text { and } \mathbf{c d}\right. \text { in parents) } \\
& =\mathbf{P}\left(\mathbf{C F}_{b} \mid \mathbf{a b}\right) \times \mathbf{P}\left(\mathrm{CF}_{\mathbf{c}} \mid \mathbf{c d}\right) \\
& \mathrm{P}\left(\mathrm{CF}_{\mathrm{ad}} \backslash \mathrm{ab} \text { and } \mathrm{cd}\right. \text { in parents) } \\
& =\mathrm{P}\left(\mathrm{CF}_{\mathrm{a}} \mid \mathbf{a b}\right) \times \mathrm{P}\left(\mathrm{CF}_{\mathrm{d}} \mid \mathbf{c d}\right) \\
& \mathbf{P}\left(\mathrm{CF}_{\mathbf{b d}} \mid \mathbf{a b} \text { and } \mathbf{c d} \text { in parents }\right) \\
& =\mathbf{P}\left(\mathrm{CF}_{\mathbf{b}} \backslash \mathbf{a b}\right) \times \mathbf{P}\left(\mathrm{CF}_{\mathbf{d}} \backslash \mathbf{c d}\right) \text {. }
\end{aligned}
$$

These calculations can also be used in the case where one of the parents carries a known mutation (for example, $\Delta \mathrm{F} 508$ associated with haplotype a) and that this mutation is present in the fetal genotype. In this case, the probability of the fetus being $\mathrm{CF}$ is equal to the probability that the haplotype contributed by the parent carries a non- $\triangle F 508 \mathrm{mu}-$ tation:

$$
\begin{aligned}
& \mathrm{P}\left(\mathrm{CF}_{\mathrm{ac}} \mid \mathbf{a b} \text { and } \mathbf{c d} \text { in parents) (when } \mathbf{a}\right. \text { is } \\
& \mathrm{CF})=\mathrm{P}\left(\mathrm{CF}_{\mathbf{c}} \mid \mathbf{c d}\right) \text {. }
\end{aligned}
$$

Finally, the risk for a further pregnancy could also be modified if the couple has a phenotypically normal child.

Although when counselling for a further pregnancy in obligate CF carriers the risk figures obtained for some genotypes are as good, or even better, than those obtained with microvillar enzyme (MVE) analysis alone, for most cases haplotype analysis should be combined with MVE in a Bayesian calculation. Haplotype data are entered as the 'prior probability' in the couple for a fetus with CF and MVE data as the 'conditional probability'. ${ }^{14}$

In the case of the sibs of a dead CF child, for whom no genetic material is available, and whose parents are negative for the $\Delta \mathrm{F} 508$ mutation, their risk of carrying a CF mutation could be calculated using the linkage disequilibrium data in table 1 and Bayes's theorem. ${ }^{141920}$ The probability that a child with a normal phenotype and whose parents are obligate carriers is a carrier can be calculated as follows:

$$
P\left(C_{a d} \mid a b \text { and } \mathbf{c d} \text { in parents }\right)
$$

$$
=\frac{\mathrm{P}\left(\mathrm{CF}_{\mathrm{ac}}\right)+\mathrm{P}\left(\mathrm{CF}_{\mathrm{bd}}\right)}{\mathrm{P}\left(\mathrm{CF}_{\mathrm{ac}}\right)+\mathrm{P}\left(\mathrm{CF}_{\mathrm{bc}}\right)+\mathrm{P}\left(\mathrm{CF}_{\mathrm{bd}}\right)} \text {. }
$$

In the case that only one of the chromosomes (for example, haplotype a) in the phenotypically normal subject is at risk of carrying a CF mutation (that is, when a person is known to have inherited at least one normal chromosome from his/her parents), the probability that that person is a carrier is:

$\mathrm{P}\left(\mathrm{C}_{\mathbf{a d}} \mid \mathbf{a b}\right.$ and $\mathbf{c d}$ in parents) (when $\mathbf{d}$ is nor$\mathrm{mal})=\mathbf{P}\left(\mathrm{CF}_{\mathbf{a}} \backslash \mathbf{a b}\right)$.

Two examples of the application of haplotype analysis in genetic counselling are presented in the figure. The index patient in pedigree $A$ is the brother of a dead CF child and we want to calculate the risk of his carrying a CF mutation. The family is negative for the $\Delta$ F508 mutation. Haplotypes are ascertained for the family, and the carrier risk of the subject is estimated according to his genotype (fa): 


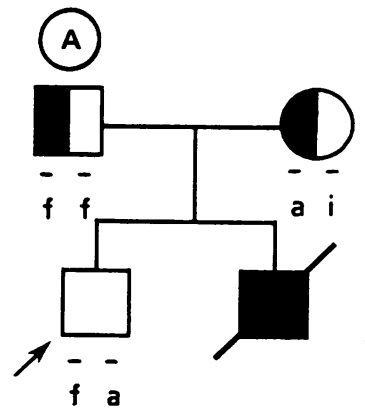

$\mathbf{P}\left(\mathrm{C}_{\mathbf{f a}}\right)$ ff and ai $)$

$$
\begin{aligned}
& =\frac{P\left(C F_{f 1}\right)+P\left(C F_{f=}\right)}{2 \times P\left(C F_{f 1}\right)+P\left(C F_{f a t}\right)} \\
& =0.50 / 0.54=0.92
\end{aligned}
$$

and is modified from 2 in 3 to 1 in $1 \cdot 1$. Another example is shown in pedigree $B$. The sister of a dead CF patient has married into the general population. The couple are negative for the $\Delta \mathrm{F} 508$ mutation, so she has not inherited $\Delta \mathrm{F} 508$ from her mother, but she may still carry the CF mutation inherited from her father. The haplotype (a) that she has inherited from her father has a high risk $(92.5 \%)$ of being a CF chromosome. On the other hand, the typing for her husband shows that he is homozygous for a haplotype (d) not present in CF chromosomes. Thus, although she has a high risk of carrying a CF mutation ( 1 in 1.1 ), the risk of the couple for CF is negligible. However, if her husband had an aa genotype, then the risk that the couple had a CF child would be:

$$
1 / 4 \times P(C n-\Delta F \mid a a) \times P\left(C F_{a} \mid a f\right)=
$$$$
1 / 4 \times 1 / 7 \cdot 2 \times 1 / 1 \cdot 1=1 / 31 .
$$

\section{LINKAGE DISEQUILIBRIUM}

The degree of association between the DNA markers studied here and CF, as measured by Yule's association coefficient, is shown in table 4. Strong allelic association was detected with all RFLPs, but the highest degree of association with CF was found with pKM.19/ScrfI, an allelic system situated $125 \mathrm{~kb}$ from the CF gene. The lower disequilibrium detected with markers which are closer to the CF gene (pKM.19/PstI and pMP6d-9/MspI) is probably the result of the variation in the allelic distribution among the normal chromosomes. pKM.19/ ScrfI also shows higher association with CF than markers not analysed here, but tested by others. ${ }^{3}$ This is true for several intragenic markers that are $\triangle F 508$ mutation and haplotype analysis in two $C F$ families ( $A$ and $B$ ) with a dead $C F$ patient. The index patient is indicated by an arrow. $-=$ non $-\Delta F 508$ mutation, $+=\Delta F 508$ mutation. Haplotypes are from table 1.

located near the major $\mathrm{CF}$ mutation, ${ }^{3}$ reflecting the influence of allelic distribution among normal chromosomes in the values of disequilibrium obtained. Data for the KM.19/ScrfI allelic system in other populations (British, Italian, and German) also show a high degree of association with CF (Ramsay, Novelli, and Stutman, personal communications). Particularly interesting is the high disequilibrium coefficient value $(A=0.80)$ obtained with pKM.19/ ScrfI in non- $\triangle F 508$ chromosomes, suggesting that other common mutations should be present in the Spanish population.

The haplotype in which $\Delta \mathrm{F} 508$ arose (a) is also the commonest haplotype $(34.2 \%)$ in the non$\Delta$ F508 CF chromosomes; this haplotype is present in only $4.3 \%$ of normal chromosomes. Thus, several mutations have arisen in the same rare haplotype. The preferential association between this uncommon haplotype and CF mutations is not well understood. We do not know what the haplotype distribution was in normal chromosomes several thousand years ago in the population in which these CF mutations occurred. It might be that haplotype a was quite common in the population in which the major CF mutations originated, but hypotheses regarding selective enhancement for mutations in this haplotype should be contemplated.

Haplotype data, in order to obtain carrier risk modifications in the cases with no DNA available from the CF index patient, should be obtained for each national population. The use of particular 
haplotype data for the different ethnic groups is still more crucial, as in the case of the Basque population in Spain, where approximately $85 \%$ of $C F$ chromosomes carry the $\Delta \mathrm{F} 508$ mutation. ${ }^{21}$

Although several intragenic polymorphisms have been identified, they are not very informative, mainly because they show strong allelic association with other markers at the CF locus. ${ }^{3}$ The markers at the D7S23 locus (XV-2c and KM.19) are still playing a relevant role in genetic diagnosis of $\mathrm{CF}^{22-24} \mathrm{~A}$ large body of data has been generated during the last three years for these markers, and recombinational events have been documented, ${ }^{25}$ although some have been withdrawn, ${ }^{26}$ with almost no recombination at the KM.19 locus. The new marker described here increases the power of linkage disequilibrium in haplotype analysis when testing cases in which one or both CF mutations have not been identified. The association between alleles at the pKM.19/Scrf I locus and CF markedly improves genetic analysis in these situations.

The data presented here are useful in our population, in which the majority of CF mutations, apart from the $\Delta \mathrm{F} 508$ deletion, are uncommon. Data on the frequency of the major CF mutation in several south European populations, and the large mutation heterogeneity found in the non- $\Delta$ F508 mutant allele pool, suggest that RFLP and haplotype analysis may be the method of choice for genetic analysis in several circumstances. This is particularly true for all the Mediterranean countries, where it is estimated that only one-third of the $75000 \mathrm{CF}$ families from this region are expected to be fully informative for mutation analysis. ${ }^{12}$ Therefore, for each population, data should be obtained and analysed in order to provide useful risk modifications in the different situations. However, it is expected that mutations covering at least $5 \%$ of $\mathrm{CF}$ chromosomes each will be detected in some populations and that mutation tests could be developed that cover a large proportion of CF cases. If this is the case, linkage disequilibrium data will not be used in the way that has been shown here. However, if the number of mutations is very high, it still might be more feasible to use RFLPs for diagnostic purposes when mutation analysis for common mutations is negative and DNA is available from the index patient.

We thank $P$ Mañà for technical assistance and $H$ Kruyer for help with the manuscript. This study was supported by the Fondo de Investigaciones Sanitarias de la Seguridad Social (90E1245) (Spain) and the Institut Catala de la Salut (Catalonia).

1 Rommens JM, Iannuzzi MC, Kerem BS, et al. Identification of the cystic fibrosis gene: chromosome walking and jumping. Science 1989;245:1059-65.
2 Riordan JR, Rommens JM, Kerem BS, et al. Identification of the cystic fibrosis gene: cloning and characterization of complementary DNA. Science 1989;245:1066-73.

3 Kerem BS, Rommens JM, Buchanan JA, et al. Identification of the cystic fibrosis gene: genetic analysis. Science 1989; 245:1073-80.

4 Lemna WK, Feldman GL, Kerem BS, et al. Mutation analysis for heterozygote detection and the prenatal diagnosis of cystic fibrosis. N Engl f Med 1990;332:291-6.

5 McIntosh I, Raeburn JA, Curtis A, Brock DJH. First-trimester prenatal diagnosis of cystic fibrosis by direct gene probing. Lancet 1989;ii:972-3.

6 Estivill X, Chillon M, Casals T, et al. $\Delta$ F508 gene deletion in cystic fibrosis in Southern Europe. Lancet 1989;ii:1404.

7 Gasparini P, Pignatti PF, Novelli G, et al. Mutation analysis in cystic fibrosis. $N$ Engl $\mathcal{F}$ Med 1990;323:62-3.

8 Cutting GR, Kash LM, Rosenstein BJ, et al. A cluster of cystic fibrosis mutations in the first nucleotide-binding fold of the cystic fibrosis conductance regulator protein. Nature 1990; 346:366-9.

9 Dean M, White MB, Amos J, et al. Multiple mutations in highly conserved residues are found in mildly affected cystic fibrosis patients. Cell 1990;61:863-70.

10 White MB, Amos J, Hsu JMC, Gerrard B, Finn P, Dean M. A frame-shift mutation in the cystic fibrosis gene. Nature 1990;344:665-7.

11 Chillon M, Nunes V, Casals $T$, et al. Distribution of the $\Delta F 508$ mutation in 194 Spanish cystic fibrosis families. Hum Genet 1990;85:396-7.

12 Novelli G, Sangiuolo F, Dallapiccola B, et al. $\Delta$ F508 gene deletion and prenatal diagnosis of cystic fibrosis in Italian and Spanish families. Prenat Diagn 1990;10:413-14.

13 Nunes V, Ramsay M, Casals T, et al. Scrf I restriction fragment length polymorphism at the D7S23 locus (probe pKM.19), closely linked to cystic fibrosis. Nucleic Acids Res 1990; 18:1318.

14 Beaudet AL, Feldman GL, Fernbach SD, Buffone GJ, O'Brien WE. Linkage disequilibrium, cystic fibrosis, and genetic counselling. Am f Hum Genet 1989;44:319-26.

15 Rosenbloom CL, Kerem BS, Rommens J, et al. DNA amplification of the XV-2c polymorphism linked to cystic fibrosis. Nucleic Acids Res 1989;17:7117.

16 Anwar R, Murray K, Hedge PJ, Smith JC, Markham AF. DNA sequence analysis of the KM.19 locus linked to cystic fibrosis. Design of new oligonucleotides to remove non-specific PCR products. Hum Genet 1990;85:319-23.

17 Nunes V, Chillon M, Lench N, Ramsay M, Estivill X. PCR detection of the pKM.19/ScrfI RFLP (D7S23), a marker closely linked to the cystic fibrosis mutation. Nucleic Acids Res 1990;18:4957.

18 Huth A, Estivill X, Grade K, et al. Polymerase chain reaction for detection of the MP6d-9/MspI RFLP, a marker closely linked to the cystic fibrosis mutation. Nucleic Acids Res 1989;17:7118.

19 Watson E, Williamson R, Brueton L, Winter R. Genetic counselling for cystic fibrosis based upon mutation/haplotype analysis. Lancet 1990;ii:190-1.

20 Strain L, Curtis A, Mennie M, Holloway S, Brock DJH. Use of linkage disequilibrium data in prenatal diagnosis of cystic fibrosis. Hum Genet 1988;80:75-7.

21 Casals T, Nunes V, Mañà P, et al. Cystic fibrosis in the Basque country: European origin of the $\Delta \mathrm{F} 508$ mutation. Am $\mathcal{F} \mathrm{Hum}$ Genet 1990;47:120A.

22 Estivill X, Farrall M, Scambler PJ, et al. A candidate for the cystic fibrosis locus isolated by selection for methylation-free islands. Nature 1987;326:840-5.

23 Estivill X, Scambler PJ, Wainwright BJ, et al. Patterns of polymorphism and linkage disequilibrium for cystic fibrosis. Genomics 1987;1:257-63.

24 Estivill X, McLean C, Nunes V, et al. Isolation of a new DNA marker in linkage disequilibrium with cystic fibrosis, situated between J3.11 (D7S8) and IRP. Am F Hum Genet 1989;44:704-10.

25 Farrall N, Wainwright BJ, Feldman GL, et al. Recombinations between IRP and cystic fibrosis. Am $\mathcal{f}$ Hum Genet 1988;43:471-5.

26 Devoto M, Ronchetto P, Romano L, Romeo G. Analysis of $\Delta$ F508 does not confirm a previously reported recombination in a cystic fibrosis family. Am $\mathcal{F}$ Hum Genet 1990;46: 1004-5. 